\title{
Aspirin alleviates denervation-induced muscle atrophy via regulating the Sirt1/PGC-1 $\alpha$ axis and STAT3 signaling
}

\author{
Qiuxian Wan $^{1,2 \#}$, Lilei Zhang ${ }^{1 \#}$, Ziwei Huang ${ }^{1 \#}$, Haiyan Zhang ${ }^{1,2}$, Jing Gu$^{2}$, Hua Xu ${ }^{3}$, Xiaoming Yang ${ }^{1}$, \\ Yuntian Shen ${ }^{1}$, Betty Yuen-Kwan Law ${ }^{4}$, Jianwei Zhu ${ }^{3}$, Hualin Sun ${ }^{1 \wedge}$ \\ ${ }^{1}$ Key Laboratory of Neuroregeneration of Jiangsu and Ministry of Education, Jiangsu Clinical Medicine Center of Tissue Engineering and Nerve \\ Injury Repair, Co-Innovation Center of Neuroregeneration, Nantong University, Nantong, China; ${ }^{2}$ Department of Medical Laboratory, School of \\ Public Health, Nantong University, Nantong, China; ${ }^{3}$ Department of Orthopedics, Affiliated Hospital of Nantong University, Nantong, China; \\ ${ }^{4}$ State Key Laboratory of Quality Research in Chinese Medicine, Macau University of Science and Technology, Macau, China \\ Contributions: Conception and design: H Sun; (II) Administrative support: H Sun, J Gu; (III) Provision of study materials or patients: Q Wan, L \\ Zhang, Z Huang, H Zhang, X Yang, J Zhu; (IV) Collection and assembly of data: Q Wan, L Zhang, Z Huang, H Zhang, H Xu, X Yang, Y Shen, J \\ Zhu; (V) Data analysis and interpretation: Q Wan, L Zhang, Z Huang, J Zhu, BY Law, X Yang, H Sun; (VI) Manuscript writing: All authors; (VII) \\ Final approval of manuscript: All authors. \\ \#These authors contributed equally to this paper. \\ Correspondence to: Dr. Hualin Sun. Key Laboratory of Neuroregeneration of Jiangsu and Ministry of Education, Nantong University, 19 Qixiu Road, \\ Nantong, China. Email: sunhl@ntu.edu.cn; Jianwei Zhu. Department of Orthopedics, Affiliated Hospital of Nantong University, 20 Xisi Road, \\ Nantong, China. Email: zhujianwei_nt@163.com.
}

Background: Our prior studies have shown that inflammation may play an important triggering role during the process of denervated muscle atrophy. The nonsteroidal anti-inflammatory drug aspirin exhibits the effect of anti-inflammatory factors. This study will investigate the protective effect of aspirin on denervated muscle atrophy and the underlying mechanism.

Methods: Mouse models of denervated muscle atrophy were established. The protective effect of aspirin $(20 \mathrm{mg} / \mathrm{kg} / \mathrm{d}$, i.p.) on denervated muscle atrophy was analyzed using the wet weight ratio of tibialis anterior (TA) muscle and muscle fiber cross-sectional area (CSA). The levels of inflammatory factors were detected using quantitative reverse transcription-polymerase chain reaction and enzyme-linked immunosorbent assay. Sirtuins1 (SIRT1)/Peroxisome Proliferator-Activated Receptor $\gamma$ Co-Activator $1 \alpha$ (PGC-1 $\alpha$ ) and Signal transducer and activator of transcription 3 (STAT3) signaling pathway and the muscle fiber type related proteins in TA muscle after denervation were analyzed by western blot assay.

Results: Intraperitoneal injection of aspirin $(20 \mathrm{mg} / \mathrm{kg} / \mathrm{d})$ effectively alleviated denervation-induced muscle atrophy. This mainly manifested as follows: The wet weight ratio of TA muscle and muscle fiber CSA of mice treated with aspirin were significantly greater compared with mice treated with normal saline. The level of myosin heavy chain (MHC) increased, and the levels of muscle specific E3 ubiquitin ligase Muscle-specific RING finger-1 (MuRF-1) and muscle atrophy F-box (MAFbx) were decreased. Mitochondrial vacuolation and autophagy were inhibited, as evidenced by reduced level of autophagy related proteins PINK1, BNIP3, LC3B and Atg7 in mice treated with aspirin compared with mice treated with saline. In addition, aspirin treatment inhibited the slow-to-fast twitch muscle fiber conversion, which were related with triggering the expression of Sirt1 and PGC-1 $\alpha$. Moreover, aspirin reduced the levels of inflammatory factors interleukin-6, interleukin-1 $\beta$ and tumor necrosis factor- $\alpha$ and decreased the activation of STAT3 signaling pathway.

Conclusions: This is the first study to find that aspirin can alleviate denervation-induced muscle atrophy and inhibit the type I-to-type II muscle fiber conversion and mitophagy possibly through regulating the STAT3 inflammatory signaling pathway and Sirt1/PGC-1 $\alpha$ signal axis. This study expands our knowledge

^ ORCID: 0000-0003-1889-1561. 
regarding the pharmacological function of aspirin and provides a novel strategy for prevention and treatment of denervated muscle atrophy.

Keywords: Peripheral nerve injury; muscle atrophy; inflammatory reaction; aspirin

Submitted Jul 22, 2020. Accepted for publication Nov 08, 2020.

doi: $10.21037 / \mathrm{atm}-20-5460$

View this article at: http://dx.doi.org/10.21037/atm-20-5460

\section{Introduction}

Peripheral nerve injury is a common clinical disease. Once the peripheral nerve is injured, the target muscle will inevitably become atrophic and fibrotic (1-3). The main strategy of clinical treatment is to promote the repair and regeneration of injured nerve and delay target muscle atrophy (4-6). Skeletal muscle atrophy will occur under the pathophysiological conditions (such as cachexia, weightlessness, and immobilization) in addition to denervation (7). Therefore, clarifying the specific molecular mechanism of muscle atrophy, looking for early triggers of muscle atrophy, and intervening before the onset of muscle atrophy may obtain better prevention and treatment effects.

To elucidate the molecular regulatory mechanism of denervated muscle atrophy, we systematically studied the process of denervated muscle atrophy by using gene chip and bioinformatics methods. For the first time, we proposed that denervated muscle atrophy can be divided into four different transcription stages: oxidative stress, inflammation, atrophy, and atrophic fibrosis. This may be explained by the fact that the target muscle loses its contractile function due to denervation, resulting in reduced blood flow perfusion of the target muscle, thus the target muscle is in a relatively hypoxic state and contributes to the formation of reactive oxygen species (ROS). If this hypoxic state cannot be improved in time, then excessive ROS will be produced, leading to the production of a large number of inflammatory factors. These inflammatory factors will further activate the inflammatory response pathway and initiate the expression of downstream atrophy related genes, which contributes to the atrophy of target muscle (3). We used antioxidants pyrroloquinoline quinone, salidroside, and isoquercitrin to counter oxidative stress reaction in the target muscle in the early stage after denervation and found that all these antioxidants significantly inhibited the oxidative stress reaction in the target muscle after denervation and delayed the atrophy process of denervated muscle $(4,8,9)$. However, it is still unclear whether the intervention on the inflammatory response in the target muscle at the early stage after denervation can achieve better prevention and treatment effect.

The nonsteroidal anti-inflammatory drug Aspirin is a widely-used drug in the clinic. Studies have shown that aspirin can inhibit the activation of Interleukin-6 (IL6)/Janus Kinase 2 (JAK2)/Signal transducer and activator of transcription 3 (STAT3) pathway induced by lipopolysaccharide in $\mathrm{BV}-2$ microglia (10), regulate the activity of c-Jun $\mathrm{N}$-terminal kinase (JNK)/STAT3 pathway to inhibit inflammation and scar formation caused by tendon injury (11), and inhibit JAK/STAT3 signaling pathway, thereby playing a role in inducing the apoptosis of tumor cells $(12,13)$. In cultured hepatocytes, Aspirin can induce the activation of Sirtuins1 (SIRT1)/Peroxisome ProliferatorActivated Receptor $\gamma$ Co-Activator $1 \alpha$ (PGC-1 $\alpha$ ) signal axis, thus affecting mitochondrial metabolism and energy utilization (14). A previous study has shown that activation of Sirt1/PGC-1 $\alpha$ pathway can evoke slow oxidative myogenic programs (15). However, the effect of Aspirin on denervated muscle atrophy has not been reported. Therefore, in this study, we first established a mouse denervated muscle atrophy model to explore the effect of Aspirin on denervated muscle atrophy and the underlying mechanism.

We present the following article in accordance with the ARRIVE reporting checklist (available at http://dx.doi. org/10.21037/atm-20-5460).

\section{Methods}

\section{Establishment of mouse models of sciatic nerve transection}

All animal operations in this study was carried out according to the recommendations of Institutional Animal Care and Use Committee of Nantong University, China. Experiments were performed under a project license (No. S20200312-003) granted by ethics board of Nantong University, in compliance with national guidelines for the 
care and use of animals. The protocol was approved by the Institutional Animal Care and Use Committee of Nantong University (No. S20200312-003). Adult male ICR mice (about $20 \mathrm{~g}$ ) provided by the Laboratory Animal Center of Nantong University were randomly divided into three groups of six animals each. Mice were intraperitoneally injected with mixed narcotics $(100 \mathrm{mg} / \mathrm{kg}$ ketamine plus $10 \mathrm{mg} / \mathrm{kg}$ xylazine). After the mice were fully anesthetized, disinfection and skin preparation were performed. A $1-\mathrm{cm}$ long incision was made at the femur of the mice. After blunt separation of the skin and muscle tissues, the sciatic nerve was exposed and a $1-\mathrm{cm}$ long sciatic nerve defect was created. After debridement and disinfection, the incision was sutured. After the surgical incisions were closed, animals were housed in temperature and humiditycontrolled SPF cages, maintained under $12 \mathrm{~h}$ light/12 h dark cycles, and allowed free access to water and food. Aspirin (20 mg/kg/d, Den + Asp group) or saline (Den group) was administrated by intraperitoneal injection after sugruy and lasted for 14 consecutive days, respectively. In the sham group (Ctrl group), the mice were subjected to similar surgical procedures but without sciatic nerve transection, then equal amount of normal saline was injected. On day 14 after surgery, all mice were killed by euthanasia, and the tibialis anterior (TA) muscles of each mouse were collected and then preserved using different methods according to experimental requirements.

\section{Real-time quantitative polymerase chain reaction}

Total RNA of skeletal muscle cells was extracted by Trizol reagent. After freezing and grinding, $1 \mathrm{~mL}$ of Trizol was added to every $50 \mathrm{mg}$ of tissue. Chloroform was added for phase separation. RNA was precipitated with isopropanol and washed with $75 \%$ ethanol solution. Then RNA was reverse-transcribed into cDNA. In a $20 \mu \mathrm{L}$ reaction volume, taking $\mathrm{cDNA}$ as a template, quantitative reverse transcription-polymerase chain reaction (RTqPCR) was performed at $37^{\circ} \mathrm{C}$ for 60 minutes using iTaq Fast SYBR Green Supermix (Bio-Rad, Hercules, CA, USA). Primers used for RT-qPCR were as follows: Mouse IL-6: CTGCAAGAGACTTCCATCCAG, AGTGGTATAGACAGGTCTGTTGG; mouse IL-1 $\beta$ : GAAATGCCACCTTTTGACAGTG, TGGATGCTCTCATCAGGACAG; mouse T N F - $\alpha$ : CA G G C G G T G C C TAT G T C T C, CGATCACCCCGAAGTTCAGTAG; mouse GAPDH AACTTTGGCATTGTGGAAGG,
ACACATTGGGGGTAGGAACA. The cycle threshold (CT value) was measured, and the relative mRNA expression was calculated by $2^{-\Delta \Delta C T}$ method (16).

\section{Western blot analysis}

The total protein of skeletal muscle was extracted by RIPA lysis buffer. The content of protein was quantified by bicinchoninic acid method. Thirty microgram of total protein per lane was loaded and then separated by SDSPAGE. After separation, all proteins were transferred onto a PVDF membrane (Millipore, Billerica, MA, USA). Then the PVDF membrane was blocked with $5 \%$ non-fat milk in Tris-buffered saline solution. After three washes with Tris-buffered saline solution, the PVDF membrane was incubated with the antibodies: mouse anti-MHC (R\&D Systems, Minneapolis, MN), rabbit anti-MAFbx, rabbit anti-PINK1, rabbit anti-ATG7, mouse anti-BNIP3, rabbit anti-LC3B, rabbit anti-Sirt1 and rabbit anti-PGC-1 $\alpha$ (Abcam, Cambridge, UK), rabbit anti-COX2, mouse antip-Jak1 (Tyr1034/1035)/Jak2 (Tyr1007/1008), rabbit antip-Stat3 (Tyr705) and rabbit anti-Stat3 (Cell Signaling technology, MA, USA), rabbit anti-MuRF-1, and mouse antitubulin (Santa Cruz, Santa Cruz, CA) at $4{ }^{\circ} \mathrm{C}$ overnight. The next day, the PVDF membrane was washed three times with Tris-buffered saline solution at room temperature and then incubated with horseradish peroxidase (HRP) conjugated secondary antibodies (Sigma-Aldrich, St. Louis, MO, USA) for 1-2 hours at room temperature. Finally, the target protein was visualized by enhanced chemiluminescence (Thermo Scientific, Park Ellisville, MO, USA) in the dark, and the relative levels of the target protein was analyzed by Image J software. The gray value obtained was further standardized based on the reference control.

\section{Immunofluorescence staining}

The TA muscle of mice was fixed with $4 \%$ paraformaldehyde. The fixed muscle tissue was dehydrated by gradient sucrose solution, and then chopped and cut into $10 \mu \mathrm{m}$ thick sections on the quick-freezing table. The sections were then placed on the adhesive slide and left overnight at room temperature for drying. The slides to be dyed were washed with PBS three times for 10 minutes and blocked with blocking solution for 1 hour. To determine the crosssectional area (CSA) of skeletal muscle fiber and the content of fast twitch muscle fiber, slices were incubated with laminin antibody (Abcam, Cambridge, UK; dilution 
$1: 200)$ and fast myosin skeletal heavy chain antibody (Abcam; dilution 1:200) at $4{ }^{\circ} \mathrm{C}$ for 12 hours, respectively. Then the slides were incubated with secondary antibody Alexa Fluor (Invitrogen Antibodies, Waltham, MA, USA) at room temperature for 60 minutes. Finally, the slides were photographed with a fluorescence microscope (Zeiss, Germany) to obtain the CSA of skeletal muscle fiber and the content of fast twitch muscle fiber.

\section{ELISA assay}

Serum interleukin-6 (IL-6), interleukin-1 $\beta$ (IL-1 $\beta$ ), and tumor necrosis factor-alpha (TNF- $\alpha$ ) were detected using an ELISA kit (Beyotime, Shanghai, China). Fresh whole blood samples were obtained and kept at room temperature for 2 hours. The upper layer of serum was separated and centrifuged at $1,000 \times \mathrm{g}$ for 10 minutes at $4{ }^{\circ} \mathrm{C}$. The lightyellow supernatant was harvested. The standard samples of different concentrations were added into the designated holes, with $100 \mu \mathrm{L}$ supernatant per hole. The holes were sealed with plastic membrane for 120-minute incubation at room temperature. The mixture of $50 \mu \mathrm{L}$ serum sample and $50 \mu \mathrm{L}$ analytical buffer was incubated for 120 minutes at room temperature. After addition of biotinylated antibody, $100 \mu \mathrm{L} /$ hole, the holes were sealed with plastic film, and the mixture was incubated for 20 minutes at room temperature in the dark. After five washes, Tris-buffered saline solution, $100 \mu \mathrm{L} / \mathrm{hole}$, was added. The holes were sealed with plastic film and the resulting mixture was incubated at room temperature for 20 minutes in the dark. When the color of the resulting mixture changed obviously, stop solution, $100 \mu \mathrm{L} / \mathrm{hole}$, was added. After slight shaking, absorbance value at $450 \mathrm{~nm}$ was immediately measured. Since the concentration of target protein is directly proportional to the absorbance at $450 \mathrm{~nm}$, the concentration of target protein can be calculated by measuring absorbance value at $450 \mathrm{~nm}$ by ELISA assay.

\section{Transmission electron microscopy (TEM) analysis}

TEM was used to observe the changes of mitochondria in skeletal muscles. The detailed procedures of TEM for muscle were previously reported $(4,17)$. Briefly, muscles were first fixed in $2.5 \%$ glutaraldehyde, subsequently were fixed in $1 \%$ osmium tetroxide. Finally, muscle sections were analyzed by TEM (Hitachi, Tokyo, Japan).

\section{Statistical analysis}

All data are expressed as mean \pm SD and were statistically analyzed using GraphPad Prism 8.0.1 software. Oneway ANOVA and Tukey's post hoc test were used for data comparison between groups. A P value of 0.05 was considered statistically significant.

\section{Results}

\section{Aspirin alleviated denervated muscle atrophy}

Aspirin is a classic antipyretic, analgesic, and antiinflammatory drug that is widely used in the clinic. To determine whether Aspirin can alleviate denervated muscle atrophy, the wet weight ratio of TA muscle and muscle fiber CSA of mice were measured in each group. The wet weight ratio of TA muscle and muscle fiber CSA of mice in the Den group were significantly lower than those in the control group. This suggested that mouse models of denervated muscle atrophy were successfully established. The wet weight ratio of TA muscle and muscle fiber CSA of mice in the Den + Asp group were significantly higher compared with the Den group. These results suggested that Aspirin can alleviate denervated muscle atrophy (Figure 1).

\section{Aspirin inbibited ubiquitin proteasome bydrolysis in denervated skeletal muscle}

Muscle specific E3 ubiquitin ligases MuRF-1 and MAFbx are highly associated with muscle atrophy and are one of the important signs of muscle atrophy (18). MHC is an important structural protein of muscle fiber and an important target protein of ubiquitin proteasome hydrolysis during muscle atrophy (19). Western blot analysis showed that the level of MHC was significantly decreased in the Den group, indicating that MHC was extensively hydrolyzed during denervated muscle atrophy. Aspirin treatment reversed the decrease of expression of MHC in denervated muscles. The expression of muscle specific E3 ubiquitin ligases MuRF-1 and MAFbx increased significantly after denervation. Aspirin significantly inhibited the increase of MuRF-1 and MAFbx expression. This result suggests that Aspirin may inhibit the decrease of MHC expression induced by denervation through inhibiting the hydrolysis of ubiquitin protein in denervated 

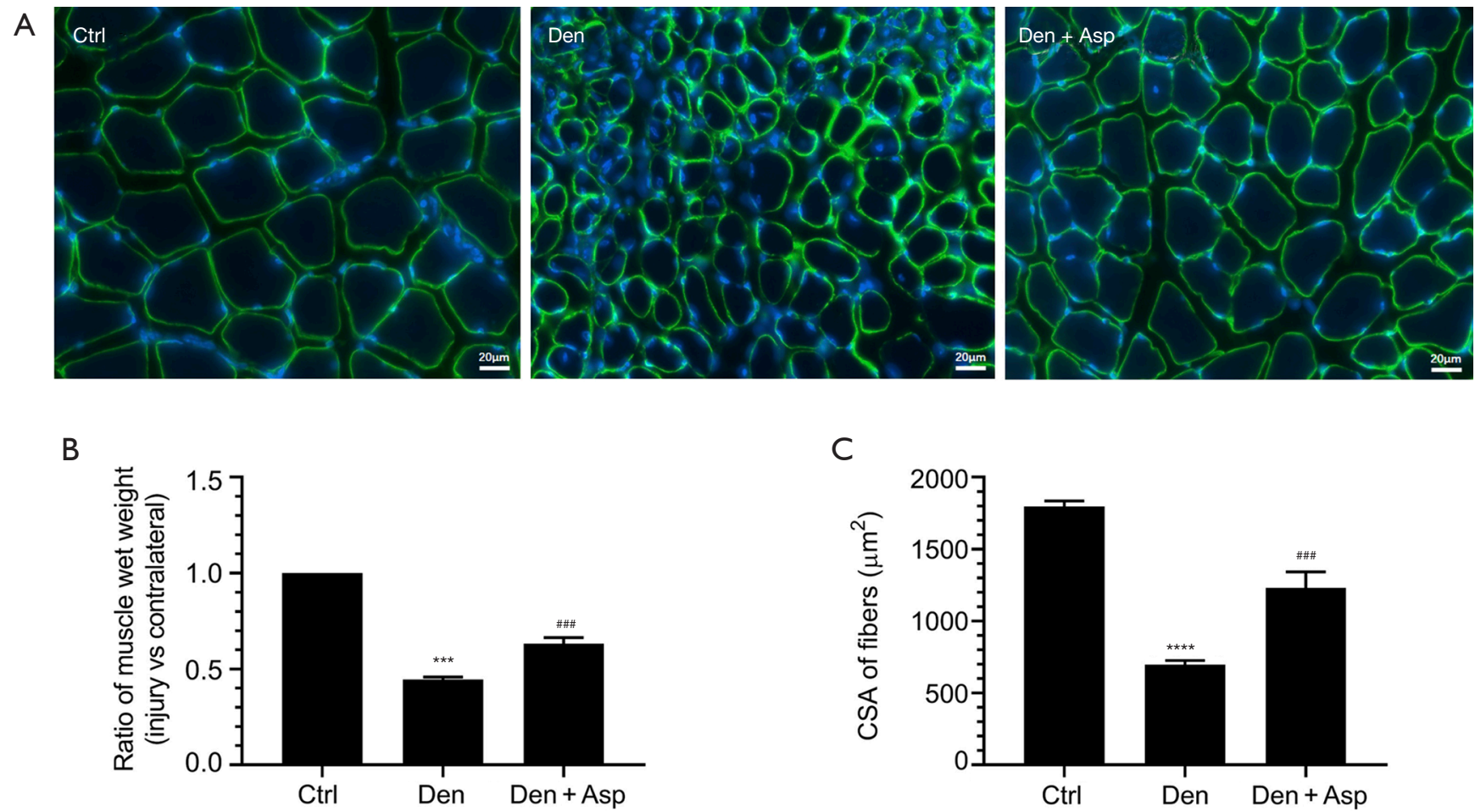

Figure 1 Aspirin alleviated denervated muscle atrophy. (A) Typical laminin staining images of muscle fiber cross-sections of the tibialis anterior (TA) muscle (bar: $20 \mu \mathrm{m}$ ); (B) statistical chart of muscle wet weight ratio of TA muscle; (C) statistical chart of cross-sectional area of TA muscle fiber. ${ }^{* * *}, \mathrm{P}<0.001$ and ${ }^{* * *}, \mathrm{P}<0.0001$ versus $\mathrm{Ctrl}$ group; ${ }^{\# \#}, \mathrm{P}<0.001$ verbs Den group.

skeletal muscle. COX2 can be induced by inflammatory factors and enhance the inflammatory response. Whether Aspirin exhibits effects on denervated muscle atrophy can be confirmed by detecting COX2 expression. Our results showed that Aspirin significantly inhibited the expression of COX2 in the TA muscle (Figure 2). These results suggested that Aspirin can alleviate denervated muscle atrophy by decreasing the expression of COX2 and further inhibiting proteasome hydrolysis.

\section{Aspirin inbibited the level of autophagy-related proteins in the denervated muscle}

In current study, vacuolar degeneration and autophagy were observed in a large number of mitochondria in the Den group, however, Aspirin could significantly inhibit mitochondrial vacuolar degeneration and autophagy (Figure $3 A$ ). Western blot assay revealed that autophagyrelated proteins PINK1, BNIP3, LC3B and ATG7 in the Den group were significantly increased. This suggests that during the process of denervated muscle atrophy, autophagy occurred in a large number of mitochondria, while Aspirin treatment significantly inhibited the increase in the expression of autophagy-related proteins PINK1, BNIP3, LC3B and ATG7 (Figure 3B). These results suggested that Aspirin alleviates denervation-induced muscle atrophy possibly, which were related to the inhibiting the autophagy of mitochondria.

\section{Aspirin decreased the expression of proinflammatory factors after denervation}

We had confirmed that denervated muscle atrophy was closely related with inflammation. qRT-PCR was used to detect the mRNA expression of IL- 6 , IL- $1 \beta$, and TNF- $\alpha$. Our results showed that the mRNA expression of IL-6, IL- $1 \beta$, and TNF- $\alpha$ in denervated skeletal muscle was significantly increased, and Aspirin injection obviously inhibited the increase of these three inflammatory factors (Figure $4 A$ ). At the same time, ELISA was also performed to measure the protein levels of IL- 6 , IL- $1 \beta$, and TNF- $\alpha$. Our results showed that the expression of IL-6, IL- $1 \beta$, and TNF- $\alpha$ in mouse models of denervated muscle atrophy was significantly increased, and Aspirin significantly decreased 
A

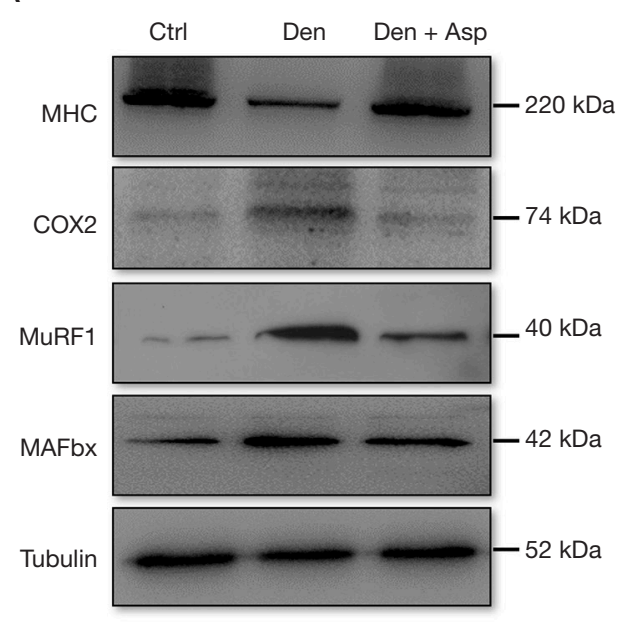

B
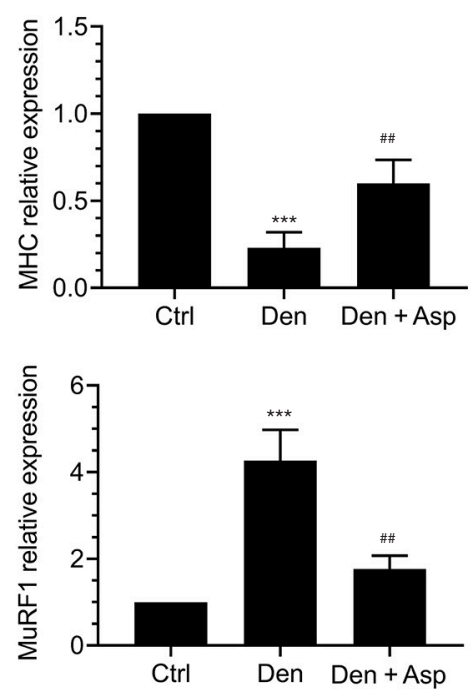
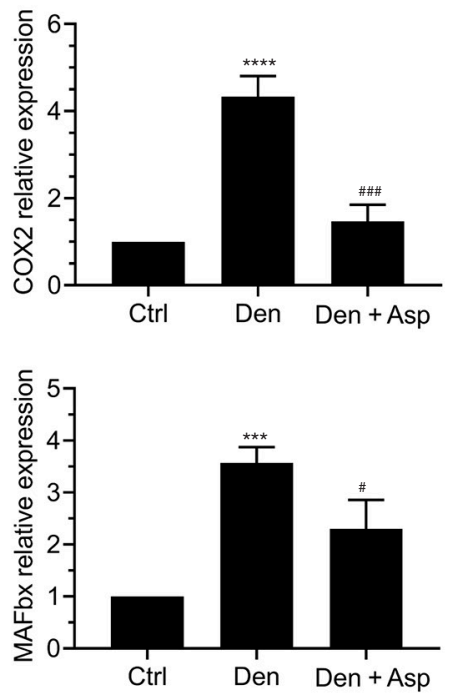

Figure 2 Aspirin inhibited the hydrolysis of ubiquitin proteasome in denervated skeletal muscle. (A) Typical western blot images of myosin heavy chain (MHC), Cyclooxygenase 2 (COX2), muscle specific E3 ubiquitin ligase Muscle-specific RING finger-1(MuRF-1) and muscle atrophy F-box (MAFbx). (B) Statistical chart of relative expression of MHC, COX2, MAFbx and MuRF1. ${ }^{* * *}, \mathrm{P}<0.001$ and ${ }^{* * * *}, \mathrm{P}<0.0001$ versus Ctrl group; ${ }^{\prime \prime}, \mathrm{P}<0.05,{ }^{\# \#}, \mathrm{P}<0.01$ and ${ }^{\# \# \#}, \mathrm{P}<0.001$, vs. Den group.

the expression of IL-6, IL-1 $\beta$, and TNF- $\alpha$ (Figure 4B). Taken together, Aspirin could decrease the expression of proinflammatory factors induced by denervation.

\section{Aspirin inbibited STAT3 signaling in the denervated skeletal muscle}

Aspirin significantly reduced the level of proinflammatory factors induced by denervation. To validate whether the downstream signaling pathways of these proinflammatory factors are also regulated, we detected JAK2/STAT3 axis, a classical downstream signaling pathway of IL-6. Our results showed that phosphorylation of JAK2 and STAT3 are increased significantly after denervation. This suggested that the STAT3 signaling pathway was significantly activated after denervation. Aspirin significantly downregulated the levels of pJAK2 and pSTAT3, which indicated Aspirin effectively inhibit the activation of STAT3 (Figure 5). We believe that Aspirin may, at least in part, alleviate denervated muscle atrophy by blocking the STAT3 signaling pathway.

\section{Aspirin inbibited fiber type conversion in denervated skeletal muscle}

Abnormal slow-to-fast twitch muscle fiber conversion was closely related with muscle atrophy $(20,21)$. We investigated the effects of Aspirin on slow-to-fast twitch muscle fiber conversion after denervation, and found that Aspirin inhibited the increase in the proportion of type II muscle fibers in denervated skeletal muscle and increased the expression of Sirt1 and PGC-1 $\alpha$ (Figure 6). The Sirt1/PGC$1 \alpha$ pathway can improve muscle fiber energy metabolism and mitochondrial function and regulate the conversion of muscle fiber types (14). These results suggested that Aspirin inhibit the conversion of type I muscle fiber to type II muscle fiber, which may be related with Sirt1/PGC-1 $\alpha$ pathway, thus alleviating skeletal muscle atrophy after denervation.

\section{Discussion}

Our prior study found that inflammation plays an important role in triggering the process of denervated muscle atrophy (3). Theoretically, anti-inflammatory drugs should be able to inhibit the inflammatory response in denervated skeletal muscle, thus playing an antiatrophic role. Therefore, we are eager to know whether anti-inflammatory drugs really have such effects. In this study, we investigated the effect of Aspirin, a classical nonsteroidal anti-inflammatory drug, on denervated muscle atrophy in mice. We found that Aspirin exhibited excellent anti-atrophic role. This is the first 
A

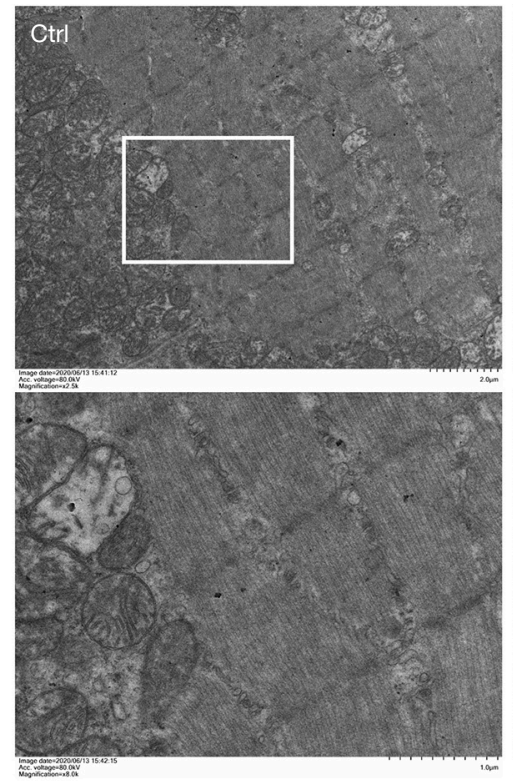

B

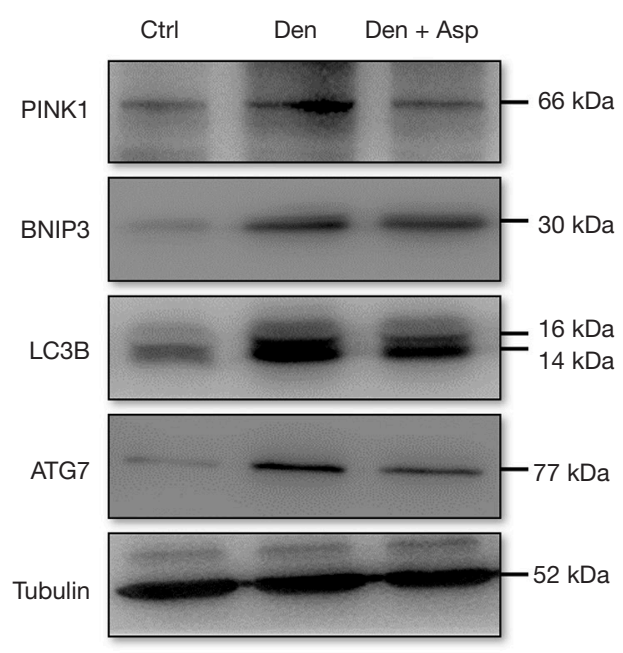

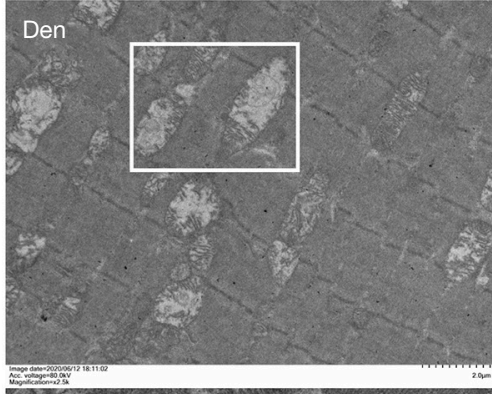
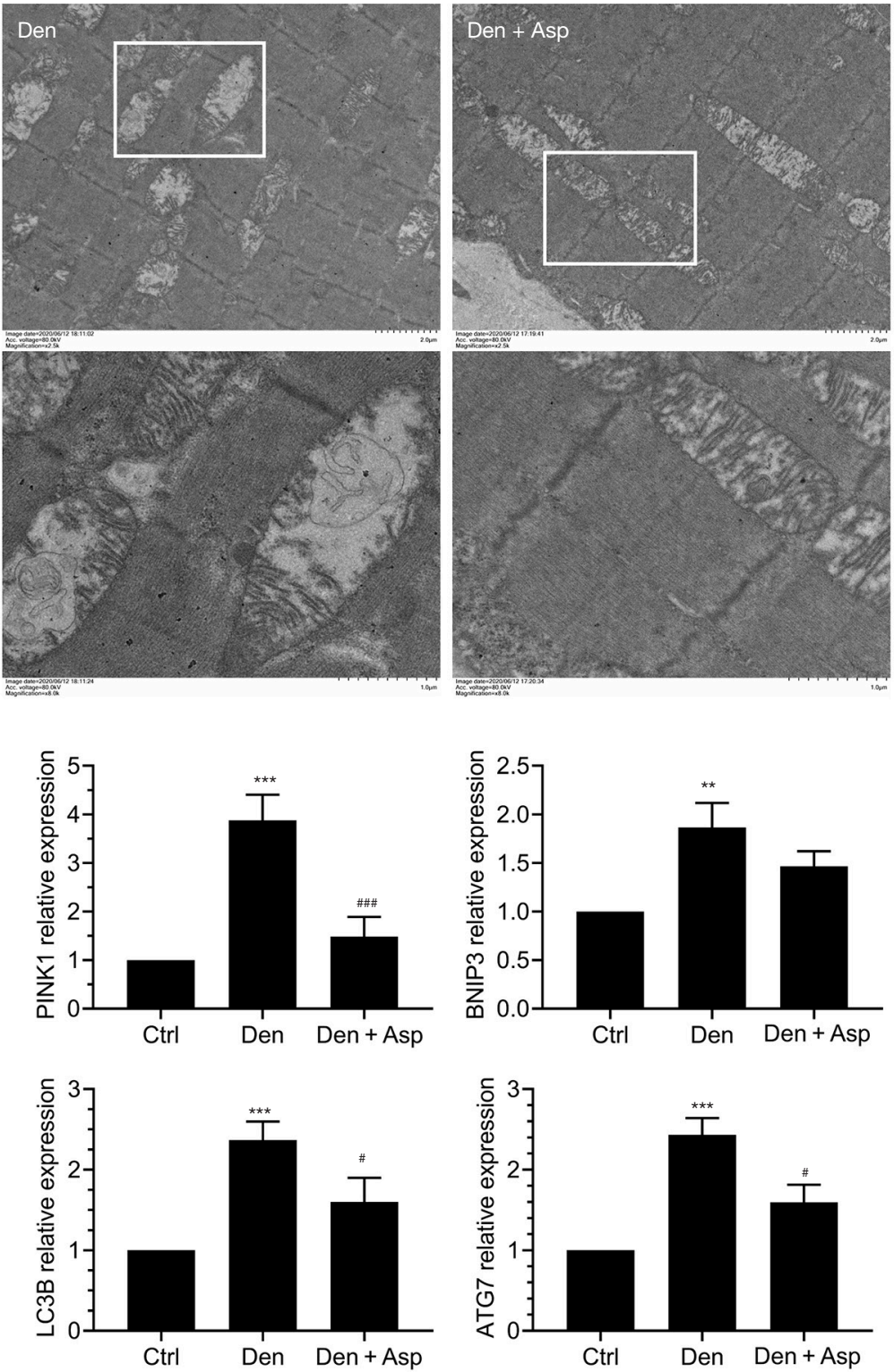

Figure 3 Aspirin inhibited mitochondrial autophagy in denervated skeletal muscle. (A) Ultrastructure of muscle fibers observed using transmission electron microscopy. (B) Western blot and quantification of the autophagy-related proteins of PTEN induced putative kinase 1 (PINK1), BCL2 interacting protein 3 (BNIP3), microtubule-associated protein 1 light chain 3 beta (LC3B) and autophagy related protein 7 (Atg7). **, $\mathrm{P}<0.01$ and ${ }^{* * *}, \mathrm{P}<0.001$ versus $\mathrm{Ctrl}$ group; ${ }^{*}, \mathrm{P}<0.05$ and ${ }^{\text {\#\#\# }}, \mathrm{P}<0.001$ versus Den group.

study to found that Aspirin can resist denervated muscle atrophy. Our study expands our knowledge regarding the pharmacological function of Aspirin.

Aspirin is a nonsteroidal anti-inflammatory drug (NSAID). It has classical antipyretic, analgesic, and antiinflammatory effects. In orthopedic treatment, Aspirin can be used to treat tendon injury and reduce the risk of tendon injury because it can inhibit inflammation and scar formation through the JNK/STAT3 signaling pathway (11). After total joint replacement, use of Aspirin for 6 successive weeks can reduce the risk of venous thromboembolism in patients (22). The above studies confirmed the classic effects of Aspirin, among which the strong anti-inflammatory effect is one of its major characteristics. There is evidence that 
A

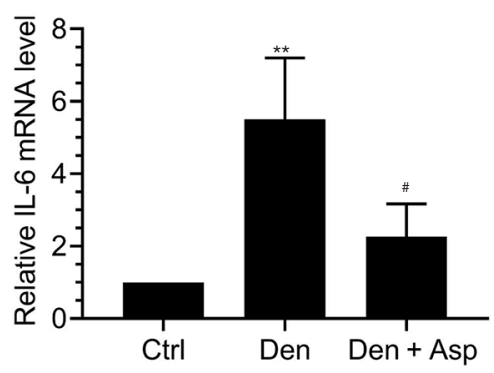

B

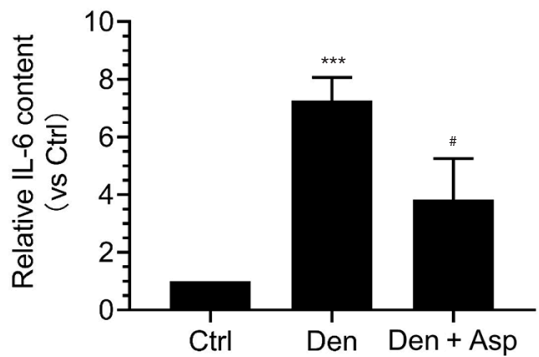

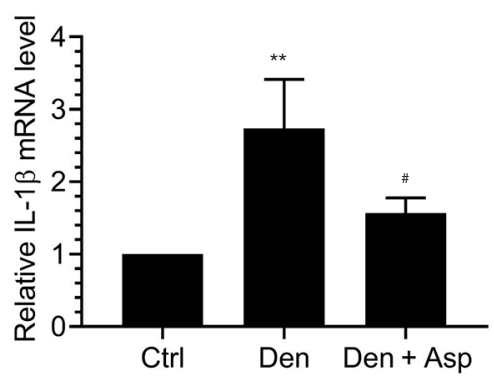
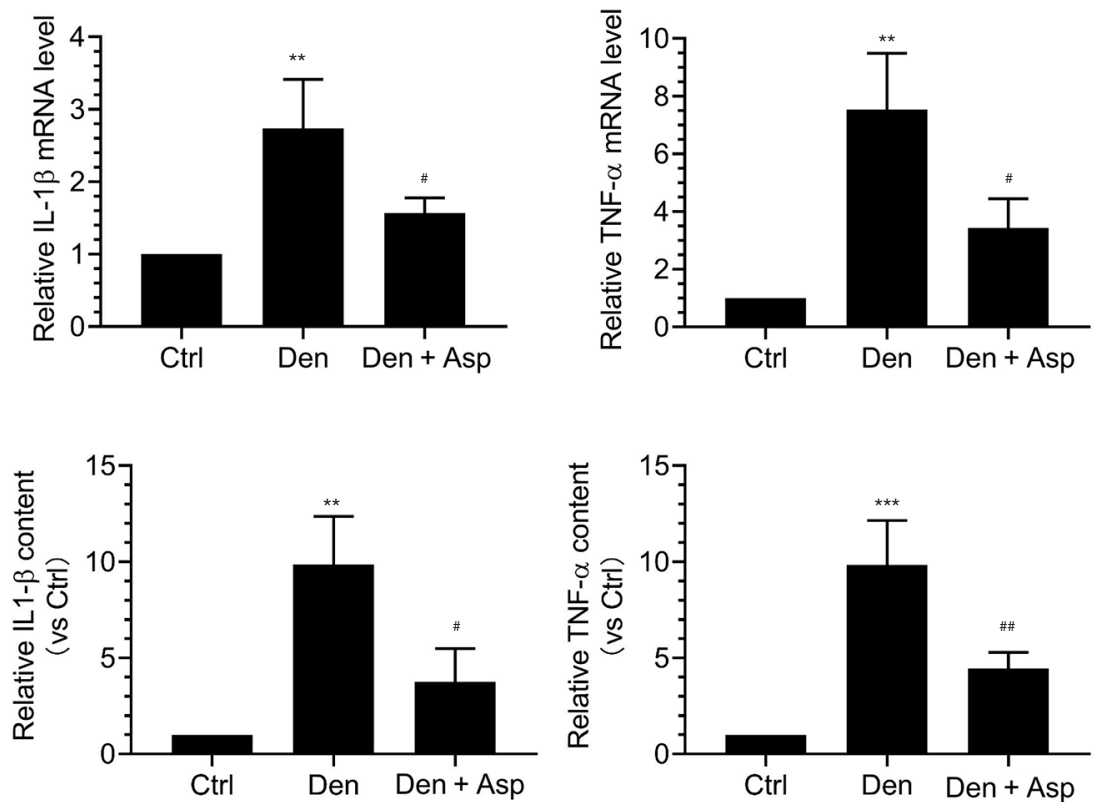

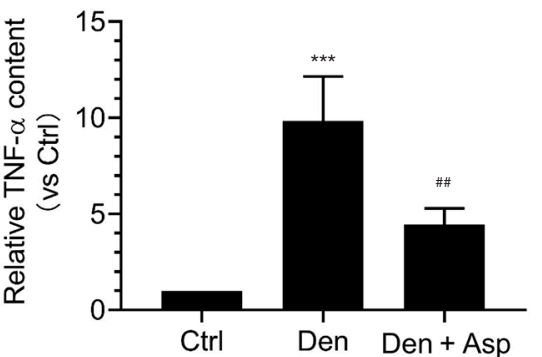

Figure 4 Aspirin decreased the expression of proinflammatory factors induced by denervation. The muscle and serum of TA were harvested for quantitative reverse transcription-polymerase chain reaction (qRT-PCR) and enzyme-linked immunosorbent assay (ELISA). (A) qRTPCR was performed to analyze the mRNA expression of proinflammatory factors IL- 6 , IL- $1 \beta$ and TNF- $\alpha$ in TA muscle of mice in each group. (B) ELISA was performed to determine the content of tumor necrosis factor- $\alpha$ (TNF- $\alpha$ ), Interleukin $-1 \beta$ (IL-1 $\beta$ ) and Interleukin- 6 (IL-6) in mice of each group. ${ }^{* *}, \mathrm{P}<0.01$ and ${ }^{* * *}, \mathrm{P}<0.001$ versus $\mathrm{Ctrl}$ group; ${ }^{*}, \mathrm{P}<0.05$ and ${ }^{\# \#}, \mathrm{P}<0.01$ versus Den group.
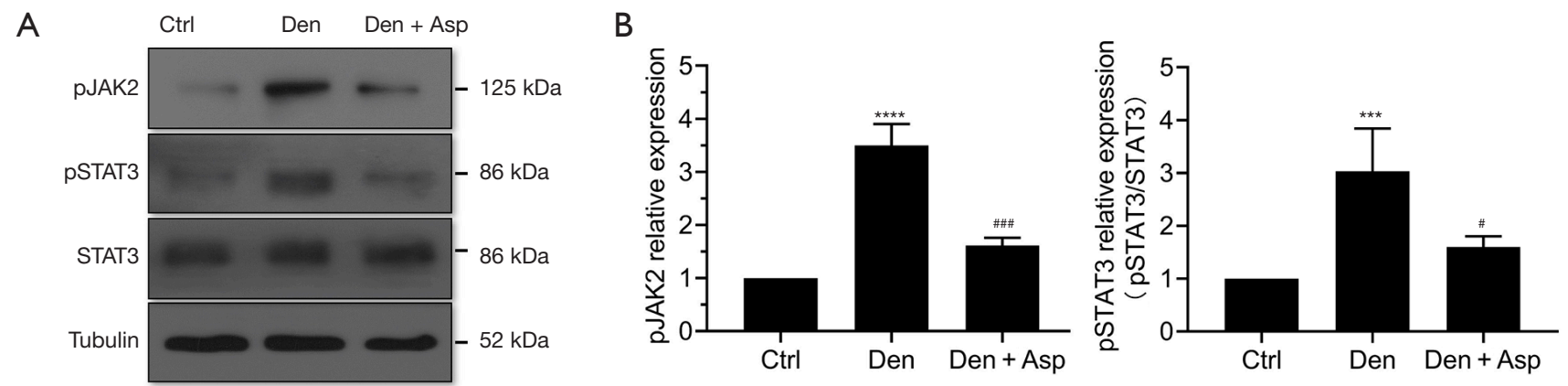

Figure 5 Aspirin regulated STAT3 signaling pathway in denervated skeletal muscle. (A) The representative western blots of signal transducer and activator of transcription 3 (STAT3), phosphorylated signal transducer and activator of transcription 3 (pSTAT3) and phosphorylated Janus kinase 2 (pJAK2). (B) Statistical chart of levels of pJAK2 and pSTAT3. ***, $\mathrm{P}<0.001$ and ${ }^{* * * *}, \mathrm{P}<0.0001$ versus $\mathrm{Ctrl}$ group;, $\mathrm{P}<0.05$ and \#\#\#, $\mathrm{P}<0.001$ versus Den group.

Aspirin can inhibit the increase of inflammatory substances induced by Chlamydia pneumonia, such as interleukin- 8 (IL8), C-reactive protein (CRP), heat shock protein 60 (HSP60) and tumor necrosis factor- $\alpha$ (TNF- $\alpha$ ) (23). In addition, studies have found that nuclear factor (NF)$\kappa \mathrm{B}$ may be an important inducer of IL-6 expression, and Aspirin can effectively inhibit the activation of NF- $\mathrm{kB}$ and then inhibit the production of IL-6 $(24,25)$.

In this study, we found that Aspirin alleviated the process of denervated muscle atrophy. Our results showed that compared with the Den group, the wet weight ratio of TA muscle and muscle fiber CSA of mice treated with Aspirin were significantly increased, and the loss of MHC content was reversed. This occurred possibly because of 
A

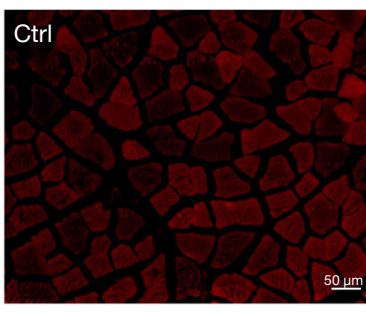

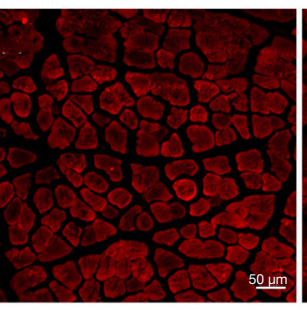
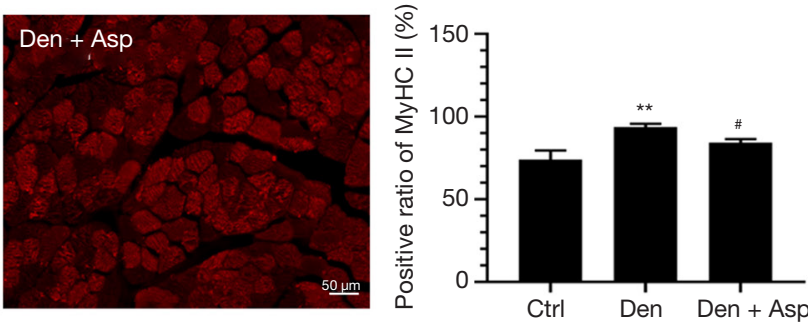

B

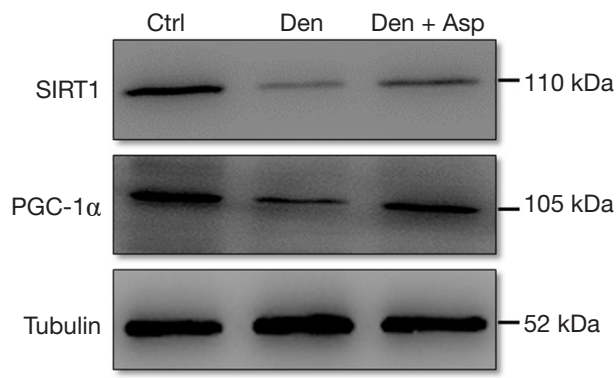

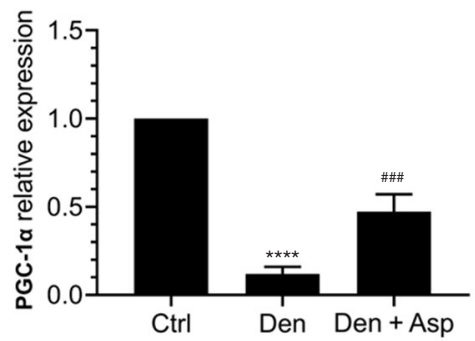

Figure 6 Aspirin inhibited the increase of the proportion of type II muscle fibers in denervated skeletal muscle. (A) Immunofluorescence staining and quantification of fast myosin skeletal heavy chain in TA muscle; (B) Western blot and quantification of sirtuin 1 (SIRT1) and proliferator-activated receptor gamma coactivator 1 -alpha $(\mathrm{PGC}-1 \alpha){ }^{* *}, \mathrm{P}<0.01$ and ${ }^{* * *}, \mathrm{P}<0.0001$ versus $\mathrm{Ctrl}$ group; ${ }^{\#}, \mathrm{P}<0.05,{ }^{\#}, \mathrm{P}<0.01$

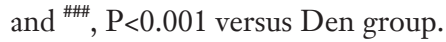

the significant inhibition of protein hydrolysis. This was also confirmed by our results: The expression of muscle specific E3 ubiquitin ligases MuRF-1 and MAFbx increased significantly during the process of denervated muscle atrophy, while Aspirin significantly inhibited the increase of MuRF-1 and MAFbx induced by denervation. That is to say, Aspirin can inhibit the hydrolysis activity of ubiquitin proteasome in the denervated skeletal muscle. The activation of autophagic-lysosomal hydrolysis system also plays an important role during the process of muscle atrophy $(26,27)$. We found that the expression of autophagy-related proteins PINK1, BNIP3, LC3B and ATG7 increased significantly during the process of denervated muscle atrophy. This suggests that autophagy lysosomal hydrolysis system was activated during the process of denervated muscle atrophy. We also found that Aspirin significantly inhibited the expression of autophagy-related proteins PINK1, BNIP3, LC3B and ATG7 in denervated skeletal muscle, suggesting that Aspirin inhibited the activity of autophagy lysosomal hydrolysis system. Studies have shown that inflammation can activate the expression of E3 ubiquitin ligases MuRF-1 and MAFbx $(28,29)$. We found that the expression of inflammatory factors IL-6, IL- $1 \beta$ and TNF- $\alpha$ in denervated skeletal muscle was significantly increased. This is consistent with our previous findings that inflammation plays an important role in triggering the process of denervated muscle atrophy (3). Aspirin can significantly inhibit the increase of IL-6, IL- $1 \beta$ and TNF- $\alpha$ in denervated skeletal muscle, and then inhibit the downstream signaling pathway. For example, once IL-6 binds to its receptor, it can activate JAK downstream and further phosphorylate STAT3. pSTAT3 can enter the nucleus as an active dimer and turn on the transcription of related functional genes (such as atrophy related genes) (12). Aspirin has been reported to reduce the production of inflammatory cytokines (IL-1 $\beta$, IL-6 and TNF- $\alpha$ ) in nucleus pulposus cells (NPCs) induced by lipopolysaccharide (LPS) (30), inhibit the JAK/STAT3 signaling pathway in patients with rheumatoid arthritis, promote the apoptosis of fibroblast-like synoviocytes and inhibit their proliferation (31). In BV-2 microglia, Aspirin inhibited the activation of IL6/JAK2/STAT3 signaling pathway induced by lipopolysaccharide (10). These reports are consistent with our results. Our study found that Aspirin can effectively reduce the phosphorylation level of JAK2 and STAT3 in denervated skeletal muscle, that is, it can significantly inhibit the activation of IL6/JAK2/STAT3 signaling pathways. These results confirm that Aspirin can inhibit the inflammatory response in denervated skeletal 


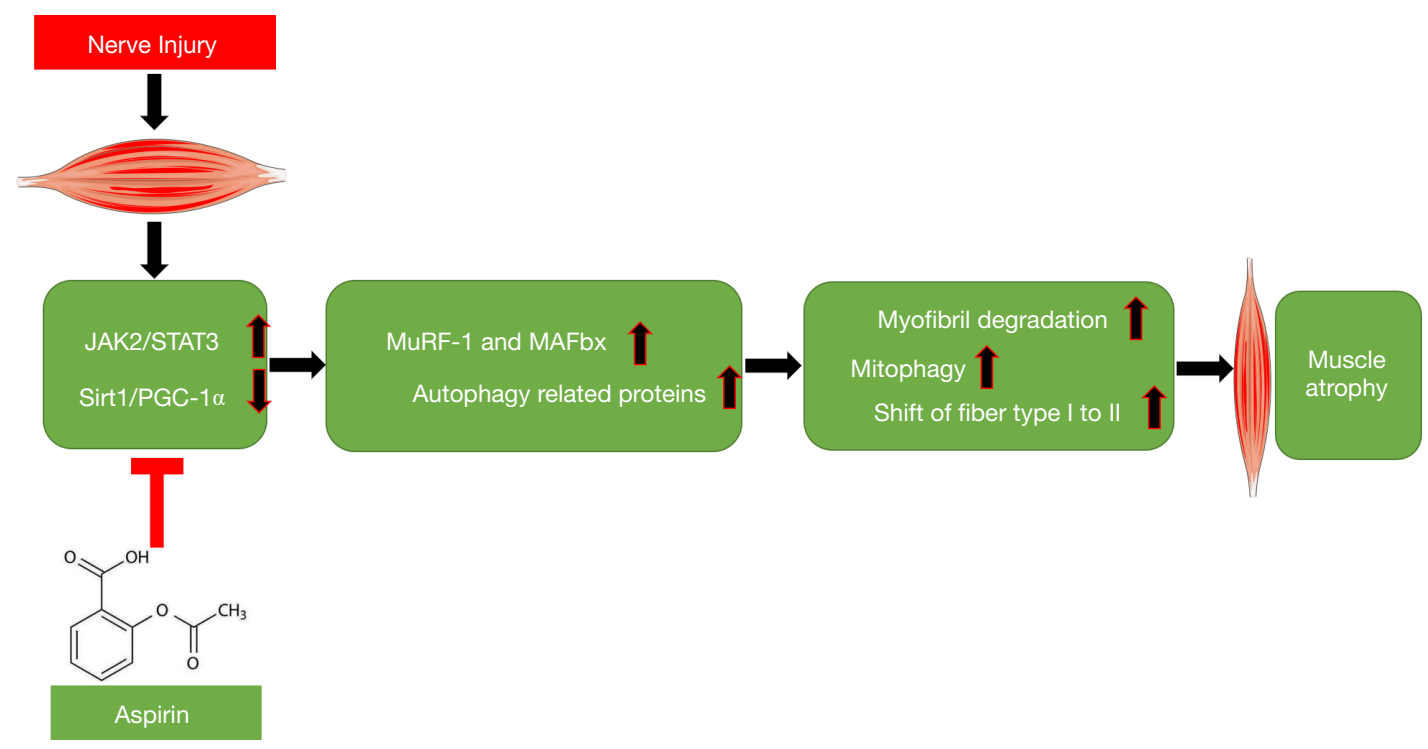

Figure 7 A schematic diagram illustrated the proposed mechanism by which Aspirin rescued peripheral nerve injury induces TA muscle atrophy.

muscle. We speculate that the protective effect of Aspirin on denervated muscle atrophy is at least partially achieved by regulating IL6/JAK2/STAT3 signaling pathway.

After the loss of innervation, the skeletal muscle gradually undergoes irreversible atrophy, which is accompanied by the increase of the proportion of type II muscle fiber (fast-switch muscle fiber), and the decrease of the proportion of type I muscle fiber (slow-switch muscle fiber), that is, the conversion from type I muscle fiber to type II muscle fiber. The increased proportion of type II muscle fiber may be a stress mechanism of muscle in response to hypoxia and inflammation, and may also be a direct cause of muscle atrophy. Sirt1 plays an important role during the process of muscle remodelling. Sirt1 can also increase the activity of PGC- $1 \alpha$, thus promoting mitochondrial biogenesis (32). PGC- $1 \alpha$ can increase the oxidative capacity of muscle fiber and cause the conversion from fast-to-slow switch muscle fiber (33). The activation of Sirt1/PGC- $1 \alpha$ signaling pathway can increase the expression of slow oxidative myogenic program, that is, Sirt1/PGC-1 $\alpha$ signaling pathway can drive the conversion of muscle fiber types and directly regulate the physiological function of skeletal muscle (15). Our study found that the expression of Sirt 1 and PGC- $1 \alpha$ decreased significantly in denervated skeletal muscle, which may be the initial factor of slow-to-fast muscle fiber conversion during the process of denervated muscle atrophy. Kamble et al. found that Aspirin induced the expression of SIRT1 and PGC- $1 \alpha$ in cultured hepatocytes, therefore affecting mitochondrial metabolism and energy utilization (14). These reports are consistent with our results. We found that Aspirin significantly reversed the downregulation of Sirt1 and PGC-1 $\alpha$ in skeletal muscle caused by denervation, thereby inhibiting the denervation-caused transformation of type I muscle fiber to type II muscle fiber. These results suggest that Aspirin delay the muscle fiber type conversion and muscle atrophy induced by denervation through Sirt1/ PGC- $1 \alpha$ signal axis.

Aspirin may also be involved in the following aspects. Our prior study found that ROS is closely related to muscle atrophy (3). Liu et al. $(30,34)$ showed that Aspirin significantly inhibited the production of ROS and oxidative stress. This phenomenon may be based on the antiinflammatory effect of Aspirin, because ROS can cause inflammation, and inflammation can also cause ROS production $(35,36)$. Aspirin can promote angiogenesis. A previous study showed that local injection of Aspirin can effectively improve the local immune environment, promote macrophage polarization, neutrophil infiltration, and increase vascular tissue regeneration (37). Whether the mechanism by which Aspirin promotes vascular regeneration is related to the protective effects of Aspirin on muscle atrophy remains poorly understood and deserves further investigation.

Taken together, this study is the first to find that Aspirin can delay denervated muscle atrophy and the conversion 
of type I muscle fiber to type II muscle fiber possibly, at least partially through regulating STAT3 signaling pathway and Sirt1/PGC-1 $\alpha$ signal axis (Figure 7). Our findings add our knowledge regarding the pharmaceutical function of the classic drug Aspirin and provides new strategies for prevention and treatment of denervated muscle atrophy.

\section{Acknowledgments}

Funding: This work was supported by the National Natural Science Foundation of China (Grant No. 82072160, $81871554,31730031,81671230$ and 81901933), the National Key Research and Development Program of China (Grant No. 2017YFA0104703), the Priority Academic Program Development of Jiangsu Higher Education Institutions (PAPD), Jiangsu College Students Innovation and Entrepreneurship Training Program (201910304033Z), the Major Natural Science Research Projects in Universities of Jiangsu Province (20KJA310012), the Natural Science Foundation of Jiangsu Province (BK20201209) and Nantong Science and Technology Innovation Program (Grant No. JC2019028).

\section{Footnote}

Reporting Checklist: The authors have completed the ARRIVE reporting checklist. Available at http://dx.doi. org/10.21037/atm-20-5460

Data Sharing Statement: Available at http://dx.doi. org/10.21037/atm-20-5460

Conflicts of Interest: All authors have completed the ICMJE uniform disclosure form (available at http://dx.doi. org/10.21037/atm-20-5460). The authors have no conflicts of interest to declare.

Ethical Statement: The authors are accountable for all aspects of the work in ensuring that questions related to the accuracy or integrity of any part of the work are appropriately investigated and resolved. All animal operations in this study were carried out according to the recommendations of Institutional Animal Care and Use Committee of Nantong University, China. Experiments were performed under a project license (No. S20200312003) granted by ethics board of Nantong University, in compliance with national guidelines for the care and use of animals. The protocol was approved by the Institutional Animal Care and Use Committee of Nantong University (No. S20200312-003).

Open Access Statement: This is an Open Access article distributed in accordance with the Creative Commons Attribution-NonCommercial-NoDerivs 4.0 International License (CC BY-NC-ND 4.0), which permits the noncommercial replication and distribution of the article with the strict proviso that no changes or edits are made and the original work is properly cited (including links to both the formal publication through the relevant DOI and the license). See: https://creativecommons.org/licenses/by-nc-nd/4.0/.

\section{References}

1. Yang Y, Ding F, Wu J, et al. Development and evaluation of silk fibroin-based nerve grafts used for peripheral nerve regeneration. Biomaterials 2007;28:5526-35.

2. Ashley Z, Sutherland H, Lanmuller H, et al. Atrophy, but not necrosis, in rabbit skeletal muscle denervated for periods up to one year. Am J Physiol Cell Physiol 2007;292:C440-51.

3. Shen Y, Zhang R, Xu L, et al. Microarray Analysis of Gene Expression Provides New Insights Into DenervationInduced Skeletal Muscle Atrophy. Front Physiol 2019;10:1298.

4. Ma $\mathrm{W}$, Zhang R, Huang Z, et al. PQQ ameliorates skeletal muscle atrophy, mitophagy and fiber type transition induced by denervation via inhibition of the inflammatory signaling pathways. Ann Transl Med 2019;7:440.

5. Fang Q, Xu T, Wu C, et al. Biotargets in neural regeneration. Biotarget 2017;1:6.

6. Huang Z, Zhu J, Ma W, et al. Strategies and potential therapeutic agents to counter skeletal muscle atrophy. Biotarget 2018:2:8.

7. Barton E, Morris C. Mechanisms and strategies to counter muscle atrophy. J Gerontol A Biol Sci Med Sci 2003;58:M923-6.

8. Huang Z, Fang Q, Ma W, et al. Skeletal Muscle Atrophy Was Alleviated by Salidroside Through Suppressing Oxidative Stress and Inflammation During Denervation. Front Pharmacol 2019;10:997.

9. Qiu J, Yang X, Wang L, et al. Isoquercitrin promotes peripheral nerve regeneration through inhibiting oxidative stress following sciatic crush injury in mice. Ann Transl Med 2019;7:680. 
10. Li WY, Li FM, Zhou YF, et al. Aspirin down Regulates Hepcidin by Inhibiting NF-kappaB and IL6/JAK2/ STAT3 Pathways in BV-2 Microglial Cells Treated with Lipopolysaccharide. Int J Mol Sci 2016;17:1921.

11. Wang Y, He G, Tang H, et al. Aspirin inhibits inflammation and scar formation in the injury tendon healing through regulating JNK/STAT-3 signalling pathway. Cell Prolif 2019;52:e12650.

12. Kim SR, Bae MK, Kim JY, et al. Aspirin induces apoptosis through the blockade of IL-6-STAT3 signaling pathway in human glioblastoma A172 cells. Biochem Biophys Res Commun 2009;387:342-7.

13. Tian Y, Ye Y, Gao W, et al. Aspirin promotes apoptosis in a murine model of colorectal cancer by mechanisms involving downregulation of IL-6-STAT3 signaling pathway. Int J Colorectal Dis 2011;26:13-22.

14. Kamble P, Selvarajan K, Aluganti Narasimhulu C, et al. Aspirin may promote mitochondrial biogenesis via the production of hydrogen peroxide and the induction of Sirtuin1/PGC-1alpha genes. Eur J Pharmacol 2013;699:55-61.

15. Huang CC, Wang T, Tung Y'T, et al. Effect of Exercise Training on Skeletal Muscle SIRT1 and PGC-1alpha Expression Levels in Rats of Different Age. Int J Med Sci 2016;13:260-70.

16. Livak KJ, Schmittgen TD. Analysis of relative gene expression data using real-time quantitative PCR and the 2(-Delta Delta C(T)) Method. Methods 2001;25:402-8.

17. Wang D, Sun H, Song G, et al. Resveratrol Improves Muscle Atrophy by Modulating Mitochondrial Quality Control in STZ-Induced Diabetic Mice. Mol Nutr Food Res 2018;62:e1700941.

18. Rom O, Reznick AZ. The role of E3 ubiquitin-ligases MuRF-1 and MAFbx in loss of skeletal muscle mass. Free Radic Biol Med 2016;98:218-30.

19. Sijts EJ, Kloetzel PM. The role of the proteasome in the generation of $\mathrm{MHC}$ class I ligands and immune responses. Cell Mol Life Sci 2011;68:1491-502.

20. Toth MJ, Callahan DM, Miller MS, et al. Skeletal muscle fiber size and fiber type distribution in human cancer: Effects of weight loss and relationship to physical function. Clin Nutr 2016;35:1359-65.

21. Lang F, Khaghani S, Turk C, et al. Single Muscle Fiber Proteomics Reveals Distinct Protein Changes in Slow and Fast Fibers during Muscle Atrophy. J Proteome Res 2018;17:3333-47.

22. Azboy I, Groff H, Goswami K, et al. Low-Dose Aspirin
Is Adequate for Venous Thromboembolism Prevention Following Total Joint Arthroplasty: A Systematic Review. J Arthroplasty 2020;35:886-92.

23. Gomez LM, Anton L, Srinivas SK, et al. Low-Dose Aspirin May Prevent Trophoblast Dysfunction in Women With Chlamydia Pneumoniae Infection. Reprod Sci 2019;26:1449-59.

24. Beetz A, Peter RU, Oppel T, et al. NF-kappaB and AP-1 are responsible for inducibility of the IL-6 promoter by ionizing radiation in HeLa cells. Int J Radiat Biol 2000;76:1443-53.

25. Liu JF, Jamieson GG, Drew PA, et al. Aspirin induces apoptosis in oesophageal cancer cells by inhibiting the pathway of NF-kappaB downstream regulation of cyclooxygenase-2. ANZ J Surg 2005;75:1011-6.

26. Cid-Díaz T, Santos-Zas I, Gonzalez-Sanchez J, et al. Obestatin controls the ubiquitin-proteasome and autophagy-lysosome systems in glucocorticoid-induced muscle cell atrophy. J Cachexia Sarcopenia Muscle 2017;8:974-90.

27. Yang X, Xue P, Liu X, et al. HMGB1/autophagy pathway mediates the atrophic effect of TGF-beta1 in denervated skeletal muscle. Cell Commun Signal 2018;16:97.

28. Valentine RJ, Jefferson MA, Kohut ML, et al. Imoxin attenuates LPS-induced inflammation and MuRF1 expression in mouse skeletal muscle. Physiol Rep 2018;6:e13941.

29. Ma W, Xu T, Wang Y, et al. The role of inflammatory factors in skeletal muscle injury. Biotarget 2018;2:7.

30. Liu Y, Lin J, Wu X, et al. Aspirin-Mediated Attenuation of Intervertebral Disc Degeneration by Ameliorating Reactive Oxygen Species In Vivo and In Vitro. Oxid Med Cell Longev 2019;2019:7189854.

31. Zhang X, Feng H, Du J, et al. Aspirin promotes apoptosis and inhibits proliferation by blocking G0/ G1 into $S$ phase in rheumatoid arthritis fibroblastlike synoviocytes via downregulation of JAK/STAT3 and NF-kappaB signaling pathway. Int J Mol Med 2018;42:3135-48.

32. Shen S, Liao Q, Liu J, et al. Myricanol rescues dexamethasone-induced muscle dysfunction via a sirtuin 1-dependent mechanism. J Cachexia Sarcopenia Muscle 2019;10:429-44.

33. Selsby JT, Morine KJ, Pendrak K, et al. Rescue of dystrophic skeletal muscle by PGC-1alpha involves a fast to slow fiber type shift in the mdx mouse. PLoS One 2012;7:e30063. 
34. Liu YX, Feng JY, Sun MM, et al. Aspirin inhibits the proliferation of hepatoma cells through controlling GLUT1-mediated glucose metabolism. Acta Pharmacol Sin 2019;40:122-32.

35. Agita A, Alsagaff MT. Inflammation, Immunity, and Hypertension. Acta Med Indones 2017;49:158-65.

36. Sho T, Xu J. Role and mechanism of ROS scavengers in

Cite this article as: Wan Q, Zhang L, Huang Z, Zhang H, Gu J, Xu H, Yang X, Shen Y, Law BY, Zhu J, Sun H. Aspirin alleviates denervation-induced muscle atrophy via regulating the Sirt1/PGC- $1 \alpha$ axis and STAT3 signaling. Ann Transl Med 2020;8(22):1524. doi: 10.21037/atm-20-5460 alleviating NLRP3-mediated inflammation. Biotechnol Appl Biochem 2019;66:4-13.

37. Shi J, Zhang X, Jiang L, et al. Regulation of the inflammatory response by vascular grafts modified with Aspirin-Triggered Resolvin D1 promotes blood vessel regeneration. Acta Biomater 2019;97:360-73. 